\title{
Efficacy of the vegetative cells of Lysinibacillus sphaericus for biological control of insecticide-resistant Aedes aegypti
}

Paula Andrea Rojas-Pinzón ${ }^{*}$ and Jenny Dussán*

\begin{abstract}
Background: The control of Aedes aegypti is usually based on chemical insecticides, but the overuse of these compounds has led to increased resistance. The binary toxin produced by Lysinibacillus sphaericus in the final stages of sporulation is used for mosquito control due to its specificity against the culicid larvae; however, it has been proved that Ae. aegypti is refractory for this toxin. Currently, there is no evidence of the use of L. sphaericus vegetative cells for mosquito biocontrol. Therefore, in this study, the vegetative cells of three $L$. sphaericus strains were assessed against a field-collected Ae. aegypti, resistant to temephos, and the reference Rockefeller strain.

Results: Vegetative cells of L. sphaericus 2362, III(3)7 and OT4b.25 produced between 90\% and 100\% of larvae mortality in the reference Rockefeller strain. Effective concentrations of each L. sphaericus strain for the four larval stages ranged from 1.4 to $2 \times 10^{7} \mathrm{CFU} / \mathrm{ml}$. Likewise, a consortium of L. sphaericus assessed against a field-collected Ae. aegypti resistant to temephos and the Rockefeller strain caused $90 \%$ of larvae mortality. Concentrations of $L$. sphaericus consortium that resulted in larvae mortality of field-collected and Rockefeller Ae. aegypti ranged from 1.7 to $2.5 \times 10^{7} \mathrm{CFU} / \mathrm{ml}$. The vegetative cells of $L$. sphaericus have no effect on the Ae. aegypti eggs and pupae.

Conclusions: The vegetative cells of $L$. sphaericus are effective against Ae. aegypti larvae, meaning that it could be used in the biological control of these mosquito species. Since the L. sphaericus consortium was effective against temephos-resistant Ae. aegypti, vegetative cells could be an alternative to overcome insecticide-resistant populations. Further studies, should be conducted to reveal the mode of action and the toxic principle of $L$. sphaericus vegetative cells.
\end{abstract}

Keywords: Lysinibacillus sphaericus, Vegetative cell, Aedes aegypti, Field-collected strain, Biological control

\section{Background}

Aedes aegypti is the vector of major tropical diseases such as dengue, chikungunya, and Zika. The control of Ae. aegypti populations is mostly based on the application of chemical insecticides. However, the overuse of compounds such as DDT, malathion, and temephos has led to the development of insecticide resistance $[1,2]$. Another issue to be considered is the negative ecological effect of these insecticides [3]. Hence, biological control

\footnotetext{
* Correspondence: pa.rojas2807@uniandes.edu.co; jdussan@uniandes.edu.co Departamento de Ciencias Biológicas, Centro de Investigaciones

Microbiológicas (CIMIC), Universidad de los Andes, Carrera 1 No. 18 A - 10, J-206, Bogotá, Colombia
} ing low levels of mosquito populations, and as such reducing the incidence of vector-borne diseases [4].

Colombia is a propitious harbour of major vectors such as Culex spp., Anopheles spp. and Aedes spp., and thus, an endemic country for the diseases transmitted by these mosquitoes [2]. Since the re-invasion of Ae. aegypti in the 1960s, prevention against the diseases transmitted by this mosquito is based on the use of chemical insecticides [5]. Although biological control of Ae. aegypti has not been implemented in Colombia, several Lysinibacillus sphaericus strains, isolated from various regions, exhibit a promising larvicidal activity to be used in mosquito control [6]. The 
toxic activity of some native strains was assessed previously against C. quinquefasciatus by Lozano and Dussán [7]. Vegetative cells of $L$. sphaericus strains OT4b.25, III(3)7, and the World Health Organization reference strain 2362 caused over 70\% mortality against third-instar larvae of $C$. quinquefasciatus and presented metal tolerance suggesting a potential use of $L$. sphaericus in the biological control of mosquitoes in polluted environments [7].

Lysinibacillus sphaericus is a bacterium with entomopathogenic activity, attributed mainly to the binary (Bin) toxin, which is produced in the final stages of sporulation [8]. After ingestion of crystals containing the two polypeptides comprising the binary toxin, these are solubilized and activated in the larval midgut where cytopathological effects take place $[9,10]$. The effects of $L$. sphaericus on mosquito larvae are well known given the specificity of the Bin toxin on Culex spp. and Anopheles spp. midgut receptors [11]. Although Ae. aegypti is closely related to these culicids, it is refractory to the $L$. sphaericus spore toxins $[12,13]$.

Other less toxic L. sphaericus proteins are the Mtx 1, 2 and 3 toxins, and the S-layer protein. The Mtx toxins are produced in the vegetative cells but are degraded by proteases at the late log phase [14]. Separately, the S-layer protein is a paracrystalline array that covers the entire surface and can be located on the vegetative cell or inside the spore [15]. In addition to its function in shape maintenance of vegetative cells, this purified protein exhibit entomopathogenic activity against Culex quinquefasciatus and Ae. aegypti larvae $[16,17]$. Recombinant Mtx toxins and S-layer protein have been assessed against $A$ e aegypti larvae $[16,18]$, but there are no reports regarding the whole vegetative cell. Recent genomic studies have revealed that some L. sphaericus strains have hemolysins and chitinases genes, which could play an important role in the entomopathogenic activity of these strains [19-21].

Resistance to commonly used insecticides such as temephos, malathion and deltamethrin have been reported in Brazil [22], Cuba [23] and Colombia [24]. Given that the emergence of resistant mosquito populations is a major concern in public health, several studies have been conducted to assess the current susceptibility of mosquito populations to chemical insecticides. In Colombia, reports indicate resistance in some areas such as Cundinamarca, where a field population of Ae. aegypti showed 1.2\% mortality at $24 \mathrm{~h}$, and consequently, it was determined to be temephos-resistant [25].

Several studies have shown the toxic activity of $L$. sphaericus crystal-spores against some mosquito vectors, but vegetative cells action has not been demonstrated. In this study, vegetative cells of L. sphaericus OT4b.25, III(3)7, and 2362 (WHO reference) strains were assessed against a temephos-resistant, the field-collected strain of Ae. aegypti and the Rockefeller reference strain.

\section{Methods}

Lysinibacillus sphaericus and Ae. aegypti strains

The L. sphaericus strains OT4b.25 and III(3)7 were isolated from beetle larvae and an oak forest soil, respectively [6]. The WHO reference strain 2362 was isolated from adult Simulium damnosum [26] and kindly donated by A. Delecluse from the Pasteur Institute in France. The larvae of the reference Ae. aegypti Rockefeller strain were kindly donated by the National Institute of Health (INS) in Bogotá, Colombia. To evaluate a field-collected strain, weekly samplings were conducted in twelve sectors of La Mesa, Cundinamarca $\left(4^{\circ} 38^{\prime} 05.9^{\prime \prime} \mathrm{N}, 74^{\circ} 27^{\prime} 45.4^{\prime \prime} \mathrm{W}\right)$ where there is a temephos-resistant Ae. aegypti population [25].

\section{Aedes aegypti maintenance}

Individuals donated by the INS were kept at $30{ }^{\circ} \mathrm{C}$ and $60-70 \%$ relative humidity under $12: 12$ light/dark photoperiod. The larvae were fed with pellet food Omega One Natural Protein Formula (OmegaSea, LLC, Painesville, USA) for cichlids, twice a week. Pupae were placed in a separate container with tap water until the adults emerged. The adults were transferred to $30 \mathrm{~cm}^{3}$ glass cages where they were continuously provided with a $10 \%$ sucrose solution to feed on. A week post-emergence, adults were deprived of sugar for $12 \mathrm{~h}$ and provided with lamb's blood (@Microgen Ltda, Bogotá, Colombia) at $37{ }^{\circ} \mathrm{C}$ through an artificial feeder. The same conditions were provided to the field-collected strain.

\section{Bioassays of $L$. sphaericus against Ae. aegypti Rockefeller strain}

Based on the $\mathrm{LC}_{50}$ found by Santana and coworkers (work submitted) for vegetative cells against Ae. aegypti, the three $L$. sphaericus strains were evaluated in a concentration of $10^{7} \mathrm{CFU} / \mathrm{ml}$ (Table 1). For the assays, the L. sphaericus strains were grown on nutrient agar (NA) at $30{ }^{\circ} \mathrm{C}$. After $15 \mathrm{~h}$, cells were recovered in $1 \mathrm{ml}$ of water, and serial dilutions were then made to calculate the bacterial titer that was added to the bioassays. To assess the effect of $L$. sphaericus strains against each mosquito stage, the tests were conducted in $30 \mathrm{ml}$ of chlorine-free water with 20 individuals from $\mathrm{F} 1$ generation and $300 \mu \mathrm{l}$ of the bacterial suspension $\left(10^{9} \mathrm{CFU} / \mathrm{ml}\right)$. Final bacterial concentrations are specified in Table 1. All the

Table 1 Lysinibacillus sphaericus vegetative cells samples tested against Aedes aegypti

\begin{tabular}{|c|c|c|c|}
\hline \multirow[b]{2}{*}{ Ae. aegypti } & \multicolumn{3}{|c|}{ Final bacterial concentration $( \pm$ SD) CFU/ml } \\
\hline & 2362 & III(3)7 & OT4b.25 \\
\hline Rockefeller & $\begin{array}{l}1.43 \times 10^{7} \\
\left( \pm 9.06 \times 10^{5}\right)\end{array}$ & $\begin{array}{l}1.52 \times 10^{7} \\
\left( \pm 6.43 \times 10^{6}\right)\end{array}$ & $\begin{array}{l}2.04 \times 10^{7} \\
\left( \pm 5.17 \times 10^{6}\right)\end{array}$ \\
\hline Field-collected & $\begin{array}{l}1.73 \times 10^{7} \\
\left( \pm 6.04 \times 10^{6}\right)\end{array}$ & $\begin{array}{l}1.79 \times 10^{7} \\
\left( \pm 5.77 \times 10^{6}\right)\end{array}$ & $\begin{array}{l}2.49 \times 10^{7} \\
\left( \pm 11.26 \times 10^{6}\right)\end{array}$ \\
\hline
\end{tabular}

Abbreviations: SD standard deviation 
trials were made in triplicate and control without bacteria was included. For the eggs, a positive control with pellet food was carried out. The number of hatched eggs, live larvae, and adults that emerged from the pupae were recorded every $24 \mathrm{~h}$ until $48 \mathrm{~h}$ for larvae and pupae, and $72 \mathrm{~h}$ for eggs.

\section{Bioassays of a L. sphaericus consortium against Ae. aegypti Rockefeller and field-collected strains}

A consortium made up by the three L. sphaericus strains was evaluated against eggs, the four larval stages, and pupae of the both Ae. aegypti strains. For each stage, the assays were conducted with the conditions mentioned previously, but the $300 \mu \mathrm{l}$ of bacterial suspension was comprised of $100 \mu \mathrm{l}$ of each L. sphaericus strain $\left(10^{9} \mathrm{CFU} / \mathrm{ml}\right)$. Final bacterial concentrations tested for each Ae. aegypti strain are listed in Table 1. The assays were run in triplicate and control without bacteria was also included. The serial dilutions used to calculate the bacterial titer and the record times for individual survival in each stage were performed as described above.

\section{Preliminary assays of hemolytic and chitinase activity}

Since hemolysins and chitinases genes were annotated in $L$. sphaericus genomes, $L$. sphaericus vegetative cells were assessed by their hemolytic and chitinase activity. Hemolysis was determined by the formation of halo on sheep blood agar (SBA). For that, L. sphaericus III(3)7, OT4b. 25 and 2362 were cultured on SBA at $30{ }^{\circ} \mathrm{C}$ for $48 \mathrm{~h}$. Chitinase activity was evaluated by the use of colloidal chitin as the only source of carbon $[27,28]$. The three $L$. sphaericus strains were grown on minimal salt medium (MSM) comprised of $\mathrm{KH}_{2} \mathrm{PO}_{4}(0.5 \mathrm{~g} / \mathrm{l}), \mathrm{Na}_{2} \mathrm{SO}_{4}$ $(2 \mathrm{~g} / \mathrm{l}), \mathrm{KNO}_{3}(2 \mathrm{~g} / \mathrm{l}), \mathrm{CaCl}_{2}(0.001 \mathrm{~g} / \mathrm{l}), \mathrm{MgSO}_{4}(1 \mathrm{~g} / \mathrm{l})$, $\mathrm{FeSO}_{4}(0.004 \mathrm{~g} / \mathrm{l})$, agar $(15 \mathrm{~g} / \mathrm{l})$ and supplemented with $4 \%$ colloidal chitin. The plates were maintained at $30{ }^{\circ} \mathrm{C}$ for $72 \mathrm{~h}$ and then stained with $2 \%$ Congo red.

\section{Statistical analysis}

To assess statistically significant differences between hatching, mortality, and adult emergence per treatment in the Ae. aegypti field-collected and Rockefeller strains, we performed ANOVA or Kruskal-Wallis non-parametric tests (when data were not normally distributed). A TukeyKramer test was performed to observe statistically significant differences between groups. A significance value of $P<0.05$ was established for all tests. All the statistical analyses were realised in R [29].

\section{Results and discussion}

Activity of L. sphaericus on Ae. aegypti Rockefeller strain

The three L. sphaericus strains showed a similar percentage of hatched eggs compared to the positive control, and these groups were statistically different from the negative control, showing that treatment had no effects on egg hatching (Kruskal-Wallis: $X^{2}=10.71, d f=4, P=0.02$ ) (Fig. 1a). In the case of L. sphaericus OT4b.25, III(3)7, and 2362 strains, once the larvae hatched, all of them died within a period of no longer than $24 \mathrm{~h}$. Although the eggs hatch when they are immersed in water with available oxygen, a food source is necessary [30,31]. Therefore, in the first hours, the bacteria could be a potential food source but when the eggs hatch, the emerging larvae die because of the larvicidal activity of $L$. sphaericus. Ovicidal activity has been reported for plants and marine sponge extracts [32, 33], but the mode of action of this compounds on eggs has not been described. Since the hatched eggs percentage was high and very similar between the three $L$. sphaericus strains and the positive control, there is no ovicidal effect against Ae. aegypti eggs. It is well known that $L$. sphaericus crystal-spores are toxic only when these are ingested; however, the mode of action of the vegetative cells is still unknown. Despite this, the results suggest that the no-feeding stages such as eggs are not susceptible to L. sphaericus.

Regarding larval instars, the three $L$. sphaericus strains caused mortality, but the efficiency varied depending on the instar (Fig. 1b-e). For the first three larvae instars, the Tukey-Kramer test indicated that there were differences between the mortality caused by each bacterial strain and the number of dead larvae in the negative control $(P<0.001)$. A mortality of $100 \%$ occurred after $24 \mathrm{~h}$ in the first-instar; and for the second- and thirdinstar, this efficiency in mortality was reached after $48 \mathrm{~h}$. In the fourth-instar, the mortality was $60 \%$ at $48 \mathrm{~h}$, but the three strains (III(3)7, OT4b.25 and 2362) all had a negative effect against this stage (Tukey-Kramer: $P=0.3$, $P=0.28, P=0.018$, respectively) (Fig. 1e).

For the pupae, we found statistically significant differences among groups (ANOVA: $F_{(3,8)}=25.21, P<0.001$ ). The percentage of emerged adults was similar for the OT4b.25 and 2362 strains, but it was different for the III(3)7 strain and the negative control (Fig. 1f). As the pupae are metabolically inactive, and fewer adults than expected emerged in the negative control, it is possible that the bacterial strains did not affect adult emergence. Reports of the effectivity of plants extracts and seaweeds on Ae. aegypti pupae suggests that these compounds contain toxic chemicals which cause mortality by contact or choking [34, 35]. As for the eggs, the results show the lack of activity of vegetative cells on pupae, suggesting that the samples tested do not act by contact on the non-feeding stages.

Activity of the $L$. sphaericus consortium on the Ae. aegypti Rockefeller and field-collected strains

Given that all the strains were very efficient against the reference Rockefeller strain, and that resistance mechanisms 

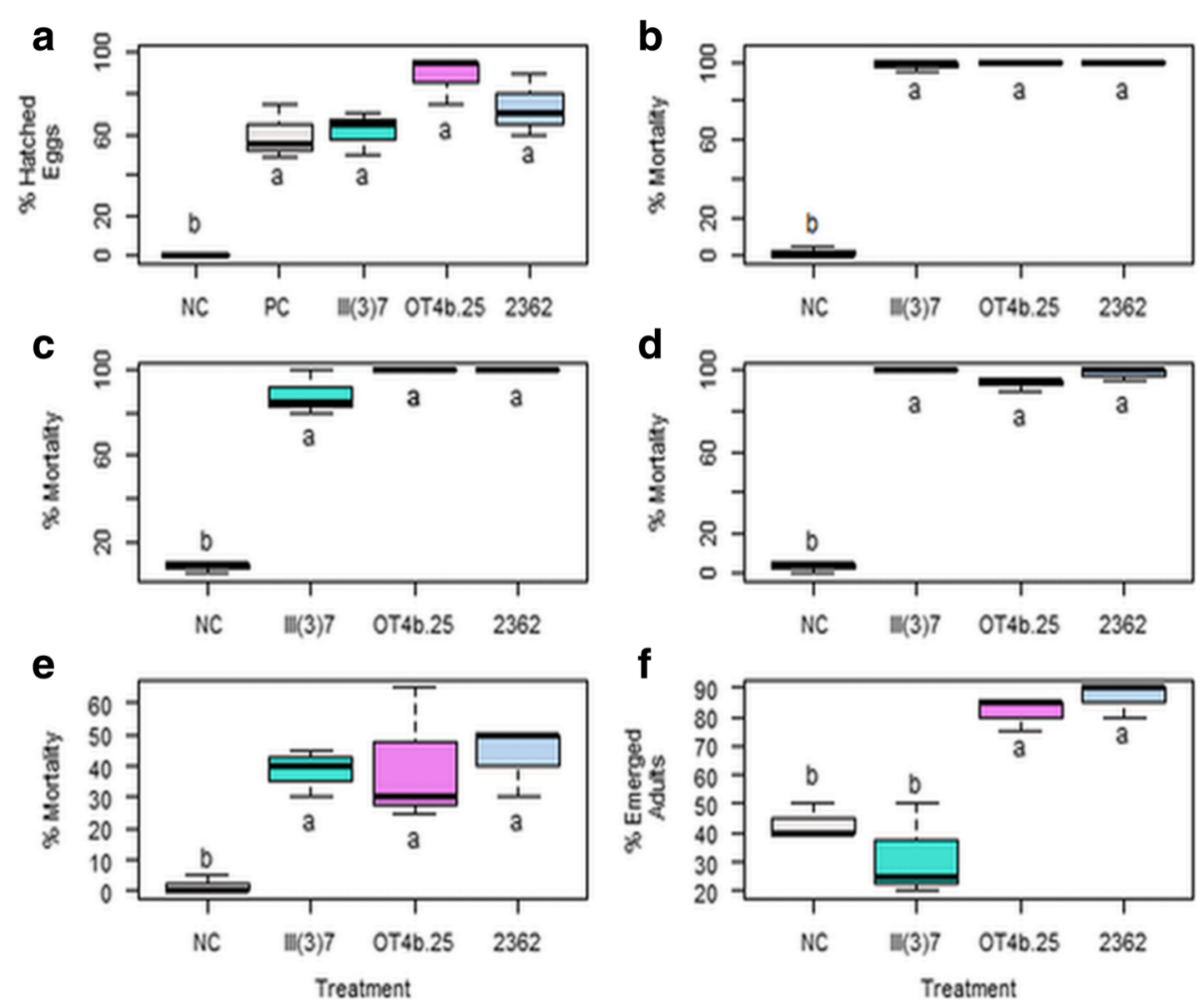

Fig. 1 Effect of L. sphaericus III(3)7, OT4b.25, and 2362 vegetative cells (between 1.4 and $2 \times 10^{9}$ CFU/ml) on the life-cycle stages of Ae. aegypti Rockefeller strain. a Percentage of eggs hatched at $72 \mathrm{~h}$, the negative control had no food and the positive control was subject to pelleted food. Percentage of larvae mortality: $\mathbf{b}$, first-instar; $\mathbf{c}$, second-instar; $\mathbf{d}$, third-instar; e, fourth-instar. $\mathbf{f}$ Percentage of emerged adults from pupae at $48 \mathrm{~h}$. Abbreviations: NC, negative control; PC, positive control. Different letters under the boxes indicate statistical significance $(P<0.05)$

are increasingly presented by the mosquitoes, a bacterial consortium was evaluated against Ae. aegypti fieldcollected and Rockefeller strains. For the eggs, the bacteria consortium seems to act similarly as individual strains because no effects on eggs hatching were seen. We hypothesised that the consortium is a primary food source, but once the eggs hatch, the first larval stage died (Fig. 2a). The statistical difference between the negative control and the percentage of eggs of both Ae. aegypti strains (Tukey-Kramer: $P=0.001, P<0.001$ ), confirm the fact that if there is no food source, the eggs do not hatch.

For all the larvae instars, the consortium had a significant effect on larva development, causing 100\% mortality at $48 \mathrm{~h}$, for the two Ae. aegypti strains (Fig. 2b-e). There were significant differences between the mortality percentage in the control and the field-collected and Rockefeller strains (Kruskal-Wallis: $\chi^{2}=7.62, d f=2, P=0.02$ ). As for the pupae (Fig. 2f), there were no statistically significant differences between the control and the treatment with the bacterial consortium in both Ae. aegypti strains (ANOVA: $\left.F_{(2,6)}=3.16, P=0.115\right)$. According to these results, both the consortium and each bacterium strain on its own had no apparent effect on adult emergence, but a higher larvicidal activity as from $24 \mathrm{~h}$.
The bacterial consortium had the same effect on the Ae. aegypti field-collected strain as on the Rockefeller strain and was more efficient than each strain evaluated separately. The better efficiency of $L$. sphaericus consortium was detected for fourth instar larvae in what there was $40 \%$ more mortality than that caused by individual strains. Since the Ae. aegypti field-collected strain is resistant to temephos and increasingly resistant populations of Ae. aegypti have appeared; the consortia of microorganisms are of great importance in the biological control of Ae. aegypti by overcoming the increase of resistance to commonly used larvicides. The organophosphate temephos is currently used due to its cost-effectiveness and the acceptance of the most vulnerable communities. However, the results herein presented suggest that $L$. sphaericus could be a better alternative when it comes to controlling Ae. aegypti populations; besides its effectivity, L. sphaericus is specific against culicids insects [36-38].

\section{Preliminary assays of hemolytic and chitinolytic activity}

The three L. sphaericus strains exhibited hemolytic activity since $24 \mathrm{~h}$, because of the formation of the transparent zone on SBA as is shown in Fig. 3a. Furthermore, due to Congo red staining is based on the differential 

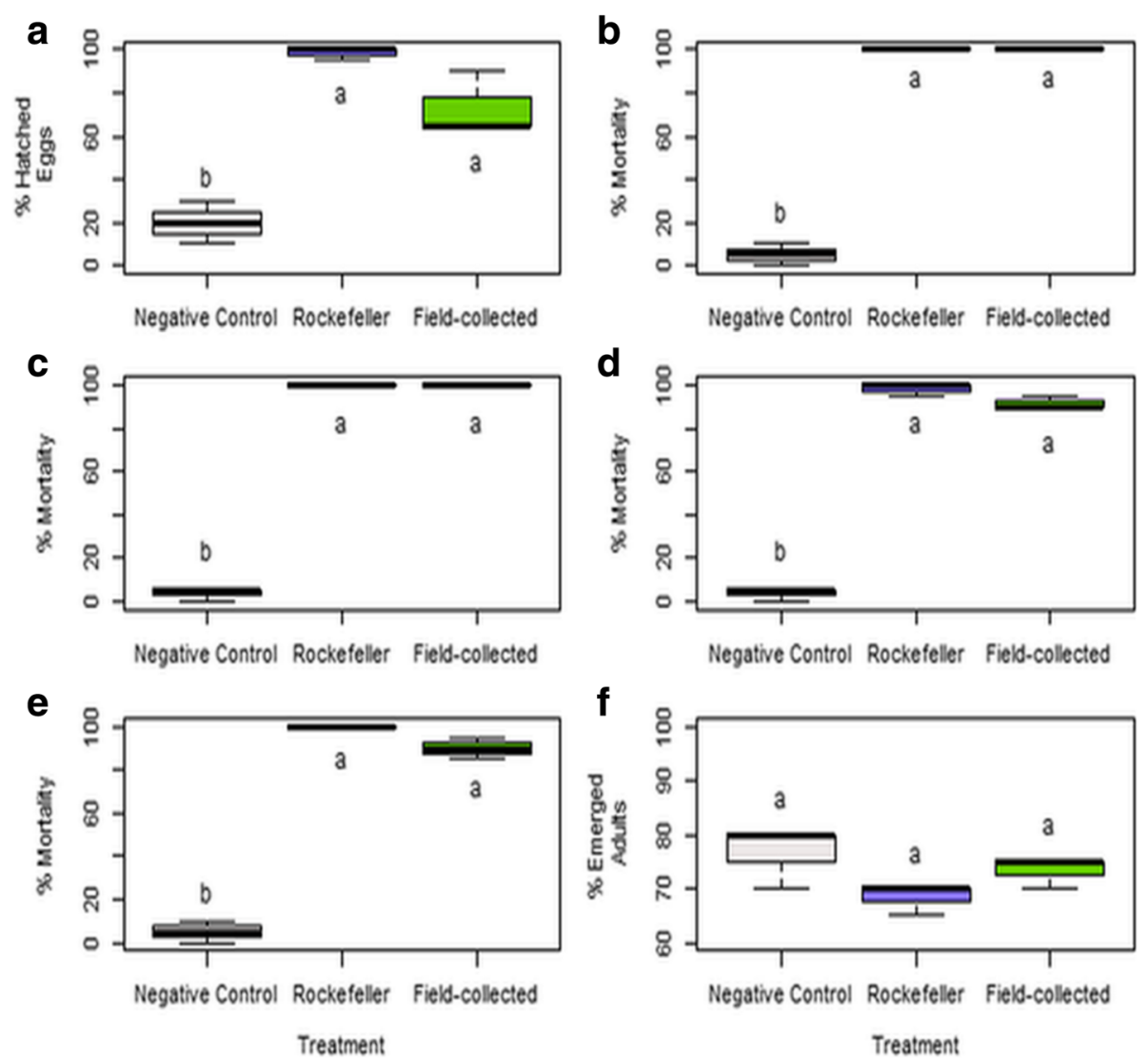

Fig. 2 Effect of a consortium of L. sphaericus vegetative cells (between 1.7 and $2.5 \times 10^{9} \mathrm{CFU} / \mathrm{ml}$ ) on the life-cycle of the Rockefeller and temephos resistant Ae. aegypti (field-collected). a Percentage of eggs hatched after $72 \mathrm{~h}$. Percentage of larvae mortality at 48 h: b, first-instar; $\mathbf{c}$, second-instar; $\mathbf{d}$, third-instar; e, fourth-instar. $\mathbf{f}$ Percentage of emerged adults from pupae at $48 \mathrm{~h}$. Different letters under the boxes indicate statistical significance $(P<0.05)$

union to chitin and there is a non-staining area around the grown of each strain, we could infer that all the strains used the colloidal chitin as carbon source Fig. 3b. In vitro assays to evaluate hemolytic and, chitinolytic activity has validated the genomic evidence regarding the presence of hemolysins and chitinases genes in some L. sphaericus strains. Although the mode of action of these toxic proteins is still unknown, the results herein presented reveals a possible key role of hemolysins and chitinases in the entomopathogenic activity of $L$.
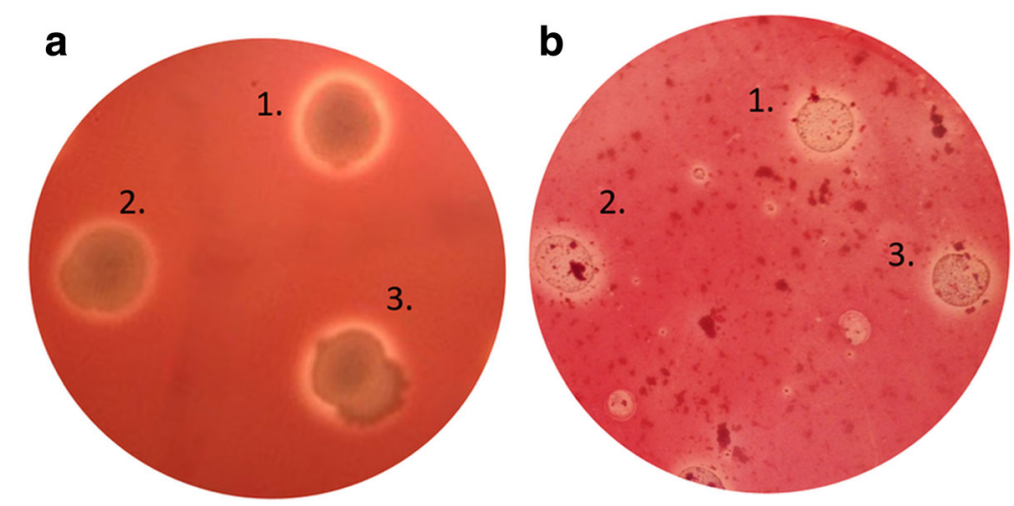

Fig. 3 In vitro evidence of the hemolytic and chitinase activity of L. sphaericus strains. a Formation of halo on SBA at $48 \mathrm{~h}$ indicate hemolysis. $\mathbf{b}$ Halo around the growth areas indicate degradation of chitin on MSM supplemented with colloidal chitin. The numbers in the figure indicate $L$. sphaericus strains: 1, III(3)7; 2, 2362; 3, OT4b.25 
sphaericus vegetative cells. As the hemolysins of other entomopathogenic bacteria [39], the hemolysins annotated in L. sphaericus could be pore-forming enzymes and thus, these might be determinant virulence factors. Likewise, as other bacterial chitinases, those of $L$. sphaericus probably play a fundamental role in the hydrolysis of chitinous structures [40].

Other less toxic proteins produced by L. sphaericus are the Mtx toxins and Cry48-Cry 49 binary protoxins. Mtx toxins are degraded into the vegetative cells [14], and Cry48-Cry 49 are toxic for C. quinquefasciatus but not for Ae. aegypti [41]. Therefore, these proteins have not been used in $L$. sphaericus-based formulations, whose effectiveness relies on the high toxicity of $L$. sphaericus crystal-spores [42, 43]. Since Ae. aeypti larvae are refractory to Bin toxins and vegetative cells exhibit a diverse collection of toxic proteins, we suggest that $L$. sphaericus vegetative cells could be used to control Ae. aegypti populations. In point of fact, we hypothesised that the effect of the vegetative cells is due to a contribution of the toxic proteins harboured in L. sphaericus vegetative cells such as hemolysins, chitinases and Slayer protein. However, the way each protein contributes to the toxicity needs to be described in future studies.

The World Health Organization suggests that instar three is the proper stage to evaluate the larvicidal effect of a new substance [44]. Our results demonstrated the effectiveness of the three bacterial strains against any of the four larval stages, suggesting that $L$. sphaericus could potentially be used as a larvicidal agent. Bacillus thuringiensis serovar. israelensis is a bacterium widely used in the control of Ae. aegypti and other culicids [45]. Even the whole crystal of Bti is used in commercial formulations for mosquito control, resistance to some single Bti toxins have been appeared in recent years [46, 47]. Therefore, the compendium of toxic proteins of $L$. sphaericus, make this bacterium a feasible solution to the resistance of mosquitoes.

Although S-layer and Mtx toxins have shown entomopathogenic activity $[16,18]$, these do not have the properties of a biological controller. Meanwhile, the vegetative cells of $L$. sphaericus can persist and recycle in the environment. L. sphaericus persistence and recycling is possible because of its ability to grow under polluted environments and resist the UV light [48]. Likewise, bacteria go through a cycle where vegetative cells germinate from a dormant spore, which makes possible the infection by the vegetative cells even if there were spores in the larval environment. Other advantages of $L$. sphaericus include safety for humans and reductions of pesticide residues in the environment [48]. Likewise, some studies have developed an easy way of industrial production of $L$. sphaericus which is a concern when a formulation is accepted for a large treatment [49]. These features together with the results herein presented make $L$. sphaericus vegetative cells a suitable biological control alternative.

\section{Conclusions}

In summary, the Ae. aegypti field-collected strain was susceptible to the vegetative cells of $L$. sphaericus which reveals the potential mosquitocidal activity of this bacterium. For a better understanding of the vegetative cells effect in Ae. aegypti larvae, future studies should be conducted to find out the role of chitinases, hemolysins and other toxic proteins genes annotated in L. sphaericus genomes. Given that the consortium was more efficient causing larvae mortality in Ae. aegypti resistant to temephos, different formulations should be assessed. The results indicate that the vegetative cells of $L$. sphaericus are a suitable alternative, which can be used to deal with insecticide resistance and to control vectors populations such as that of Ae. aegypti.

\section{Abbreviations}

Bin: Binary toxin; BSA: Blood sheep agar; INS: National institute of health; MSM: Minimal salt medium; NA: Nutrient agar

\section{Acknowledgments}

We are grateful to the National Institute of Health (INS) in Bogotá, Colombia, for the donated individuals, and to Marlon Salgado and Nestor Pintor for collection of the field-strain. Also, we want to thank to Camilo Andrés Gómez-Garzón and Maria Alejandra Hernandez for the critical review of the manuscript and to Douglas David Waters for the language corrections.

\section{Funding}

This study was funded by the Research Fund at the School of Science at Universidad de los Andes and the Microbiological Research Center (CIMIC).

\section{Availability of data and materials}

The data generated and the material used during the current study are available from the corresponding author on reasonable request.

Authors' contributions

PAR carried out all experiments, analysed the data and was a major contributor in writing the manuscript. JDG contributed with the interpretation of results and critically reviewed the manuscript. Both authors read and approved the final manuscript.

Competing interest

The authors declare that they have no competing interests.

Consent for publication

Not applicable.

Ethics approval and consent to participate Not applicable.

\section{Publisher's Note}

Springer Nature remains neutral with regard to jurisdictional claims in published maps and institutional affiliations.

Received: 27 December 2016 Accepted: 3 May 2017

Published online: 10 May 2017

\section{References}

1. Maestre-Serrano R, Gomez-Camargo D, Ponce-Garcia G, Flores A. Susceptibility to insecticides and resistance mechanisms in Aedes aegypti from the Colombian Caribbean Region. Pestic Biochem Phys. 2014;116:63-73. 
2. Ocampo C, Salazar-Terreros M, Mina N, McAllister J, Brogdon W. Insecticide resistance status of Aedes aegypti in 10 localities in Colombia. Acta Trop. 2011;118:37-44.

3. Lin $Y$, Tsen $W$, Tien N, Luo Y. Biochemical and molecular analyses to determine pyrethroid resistance in Aedes aegypti. Pestic Biochem Phys. 2013;107:266-76.

4. WHO. Dengue. Guidelines for the diagnosis, treatment, prevention and control. 2009. http://www.who.int/tdr/publications/documents/denguediagnosis.pdf Accessed 12 Apr 2017.

5. Urdaneta-Marquez L, Failloux AB. Population genetic structure of Aedes aegypti, the principal vector of dengue viruses. Infect Genet Evol. 2011;11:253-61.

6. Dussán J, Andrade DR, Lozano LC, Vanegas S. Caracterización fisiológica y genética de cepas nativas de Bacillus sphaericus. Rev Colomb Biotecnol. 2002;4:89-99.

7. Lozano L, Dussán J. Metal tolerance and larvicidal activity of Lysinibacillus sphaericus. World J Microb Biot. 2013:29:1383-89.

8. Berry C. The bacterium, Lysinibacillus sphaericus, as an insect pathogen. J Invertebr Pathol. 2012;109:1-10.

9. Silva MH, Peixoto C. Immunocytochemical localization of the Bacillus sphaericus binary toxin components in Culex quinquefasciatus (Diptera: Culicidae) larvae midgut. Pestic Biochem Phys. 2003;77:138-46.

10. Lekakarn H, Promdonkoy B, Boonserm P. Interaction of Lysinibacillus sphaericus binary toxin with mosquito larval gut cells: binding and internalization. J Invertebr Pathol. 2015;132:125-31.

11. Charles JF, Silva-Filha MH, Nielsen-LeRoux C, Humphreys MJ, Berry C. Binding of the 51- and 42-kDa individual components from the Bacillus sphaericus crystal toxin to mosquito larval midgut membranes from Culex and Anopheles sp. (Diptera: Culicidae). FEMS Microbiol Lett. 1997;156:153-59.

12. Nielsen-Leroux C, Charles JF. Binding of Bacillus sphaericus binary toxin to a specific receptor on midgut brush-border membranes from mosquito larvae. Eur J Biochem. 1992;210:585-90.

13. Ferreira LM, Romao TP, de Melo-Melo OP, Neves MH, Silva L. The orthologue to the Cpm1/Cqm1 receptor in Aedes aegypti is expressed as a midgut GPI-anchored a-glucosidase, which does not bind to the insecticidal binary toxin. Insect Biochem Molec. 2010;40:604-10.

14. Thanabalu T, Porter AG. Efficient expression of a 100-kilodalton mosquitocidal toxin in protease-deficient recombinant Bacillus sphaericus. Appl Environ Microb. 1995;61:4031-36.

15. Peña G, Miranda-Ríos J, de la Riva G, Pardo-López J, Soberón M, Bravo A. A Bacillus thuringiensis S-Layer Protein Involved in Toxicity against Epilachna varivestis (Coleoptera: Coccinellidae). Appl Environ Microbiol. 2006;72:353-60.

16. Allievi MC, Palomino MM, Prado M, Lanati L, Ruzal SM, Sánchez-Rivas C. Contribution of S-Layer proteins to the mosquitocidal activity of Lysinibacillus sphaericus. Plos One. 2014;9:1-9.

17. Lozano L, Ayala J, Dussán J. Lysinibacillus sphaericus S-layer protein toxicity against Culex quinquefasciatus. Biotechnol Lett. 2011;33:2037-41.

18. Rungrod A, Tjahaja NK, Soonsanga S, Audtho M, Promdonkoy B. Bacillus sphaericus Mtx1 and Mtx2 toxins co-expressed in Escherichia coli are synergistic against Aedes aegypti larvae. Biotechnol Lett. 2009;31:551-55.

19. Rey A, Silva L, Dussán J. Complete genome sequence of the larvicidal bacterium Lysinibacillus sphaericus strain OT4b.25. Genome Announc. 2016:4:3.

20. Rey A, Silva L, Dussán J. Complete genome sequencing and comparative genomic analysis of functionally diverse Lysinibacillus sphaericus III(3)7. Genom Data. 2016;9:78-86.

21. Hernandez-Santana MA, Gómez-Garzón C, Dussán J. Complete genome sequence of Lysinibacillus sphaericus WHO reference strain 2362. Genome Announc. 2016;4:3.

22. Lima JB, Da-Cunha MP, Da Silva RC, Garlado AK, Soares SS, Aparecida B, et al. Resistance of Aedes aegypti to organophosphates in several municipalities in the state of Rio de Janeiro and Espirito Santo. Brazil Amj Trop Med Hyg. 2003;68:329-33.

23. Bisset JA, Magdalena Rodriguez M, Fernandez D, Perez O. Status of resistance to insecticides and resistance mechanisms in larvae from Playa municipality collected during the intensive campaign against Aedes aegypti in Havana City, 2001-2002. Rev Cubana Med Trop. 2004;56:61-6.

24. Fonseca-González I, Quiñones ML, Lenhart A, Brogdon WC. Insecticide resistance status of Aedes aegypti (L.) from Colombia. Pest Manag Sci. 2011;67:430-37.

25. Santacoloma L, Chaves B, Brochero HL. Estado de la susceptibilidad de poblaciones naturales del vector del dengue a insecticidas en trece localidades de Colombia. Biomedica. 2012;32:333-43.

26. Weiser J. A mosquito-virulent Bacillus sphaericus in adult Simulium damnosum from northern Nigeria. Microbiol. 1984;139:57-60.
27. Thiagarajan V, Revathi R, Aparanjini K, Sivamani P, Girilal M, Priya CS, Kalaichelvan P. Extra cellular chitinase production by Streptomycessp. PTK19 in submerged fermentation and its lytic activity on Fusarium oxysporum PTK2 cell. Wall. Int J Curr Sci. 2011;1:30-44.

28. Soler J, Gilchrist E, Pérez JC. Evaluación de microorganismos con potencial de promoción de crecimiento vegetal y biocontrol de Spongospora subterránea. Rev Colomb Biotecnol. 2012;14:157-70.

29. $\mathrm{R}$ Core Team. R: A language and environment for statistical computing. $\mathrm{R}$ Foundation for Statistical Computing. 2015. https://www.r-project.org/. Accessed 8 Aug 2016.

30. Wong J, Stoddard ST, Astete H, Morrison A, Scott T. Oviposition site selection by the Dengue vector Aedes aegypti and its implications for Denque control. Plos Neglect Trop D. 2011:5:1-12.

31. Levi T, Ben-Dov E, Shahi P, Borovsky D, Zaritsky A. Growth and development of Aedes aegypti larvae at limiting food concentrations. Acta Trop. 2014;133:42-4.

32. Munusamy RG, Appadurai DR, Kuppusamy S, Michael GP, Savarimuthu I. Ovicidal and larvicidal activities of some plant extracts against Aedes aegypti L. and Culex quinquefasciatus Say (Diptera: Culicidae). Asian Pac J Trop Dis. 2016;6:468-71.

33. Reegan AD, Kinsalin AV, Paulraj MG, Ignacimuthu S. Larvicidal, ovicidal and repellent activities of marine sponge Cliona celata (Grant) extracts against Anopheles stephensi Liston (Diptera: Culicidae). Asian Pac J Trop Med. 2015;8:29-34.

34. Pereira $L$, Tejo $M$, Barbosa E. Bioactivity of plant extracts on the larval and pupal stages of Aedes aegypti (Diptera, Culicidea). Rev Soc Bras Med Tro. 2013:46:420-25.

35. Valentina J, Poonguzhali TV, Josmin LL. Mosquito larvicidal and pupicidal activity of seaweed extracts against Aedes aegypti, Anopheles stephensi and Culex quinquefasciatus. Int J Mosq Res. 2015;2:54-9.

36. Charles JF, Nielson-LeRoux C, Delecluse A. Bacillus sphaericus toxins: molecular biology and mode of action. Annu Rev Entomol. 1996;41:451-72.

37. Berry C, Hindley J, Ehrhardt AF, Grounds T, de Souza I, Davidson EW. Genetic determinants of host ranges of Bacillus sphaericus mosquito larvicidal toxins. J Bacteriol. 1993;175:510-18.

38. Mulla MS, Darwazeh HA, Davidson EW, Dulmage HT, Singer S. Larvicidal activity and field efficacy of Bacillus sphaericus strains against mosquito larvae and their safety to non-target organisms. Mosq News. 1984;44:336-42.

39. Jurat-Fuentes J, Jackson TA. Bacterial entomopathogens. In: Vega FE, Kaya HK, editors. Insect Pathology. 2nd ed. London: Academic; 2012. p. 265-349.

40. Frederiksen R, Paspaliari DK, Larsen T, Storgaard BG, Larsen MH, Ingmer H, et al. Bacterial chitinases and chitin-binding proteins as virulence factors. Microbiol. 2013:159:833-47.

41. Jones GW, Wirth MC, Monnerat RG, Berry C. The Cry48Aa-Cry49Aa binary toxin from Bacillus sphaericus exhibits highly restricted target specificity. Environ Microbiol. 2008;10:2418-24.

42. Brown ID, Watson TM, Carter J, Purdie DM, Kay BH. Toxicity of VectoLex (Bacillus sphaericus products) to selected Australian mosquito and nontarget species. J Econ Entomol. 2004;97:51-8.

43. Fillinger U, Knols BGJ, Becker N. Efficacy and efficiency of new Bacillus thuringiensis var. israelensis and Bacillus sphaericus formulations against Afrotropical anophelines in Western Kenya. Trop Med Int Health. 2003;8:37-47.

44. WHO. Guidelines for laboratory and field testing of mosquito larvicides. 2005 http://apps.who.int/iris/bitstream/10665/69101/1/WHO_CDS_ WHOPES_GCDPP_2005.13.pdf. Accessed 14 Apr 2017.

45. Savini V, Fazii P. Bacillus thuringiensis Insecticide Properties. In: Savini, editor. The diverse faces of Bacillus cereus. London: Academic Press; 2016. p. 139-55.

46. Tetreau G, Bayyareddy K, Jones CM, Stalinski R, Riaz MA, Paris M, et al. Larval midgut modifications associated with Bti resistance in the yellow fever mosquito using proteomic and transcriptomic approaches. BMC Genomics. 2012;13:248.

47. Paris M, Marcombe S, Coissac E, Corbel V, David JP, Despres L. Investigating the genetics of Bti resistance using mRNA tag sequencing: application on laboratory strains and natural populations of the dengue vector Aedes aegypti. Evol Appl. 2013;6:1012-27.

48. Poopathi S, Abidha S. Mosquitocidal bacterial toxins (Bacillus sphaericus and Bacillus thuringiensis serovar israelensis): mode of action, cytopathological effects and mechanism of resistance. J Physiol Pathophysiol. 2010;1:22-38.

49. Poopathi S, Kumar KA, Kabilan L, Sekar V. Development of low-cost media for the culture of mosquito larvicides, Bacillus sphaericus and Bacillus thuringiensis serovar. israelensis. World J Microb Biot. 2002;18:209-16. 